\title{
ELECTRONIC COMMERCE WITH XML/EDI IN VIRTUAL ENTERPRISES
}

\author{
A. Luís Osório ${ }^{1 *}$, Pierre Gibon ${ }^{2}$, Manuel M. Barata ${ }^{1^{*}}$ \\ ${ }^{I}$ ESTEC, Estudos e Tecnologias da Informação, \{lo, mmb\}@estec.pt, PORTUGAL \\ ${ }^{2}$ ILLICOM, Informatique, illicom@calvanet.calvacom.fr. FRANCE
}

\begin{abstract}
The Extensible Markup Language (XML) is getting a crescent adherence to represent information/data exchanged between systems, not only in the Internet domain but also to support interoperability among heterogeneous enterprise applications. The EDI community is playing a major role, contributing to establish an effective electronic commerce among SMEs with the adoption of $X M L$ and related standards in conjunction with the normalized semantics that de facto supports the EDI, as it is done nowadays. This paper discusses the advantages that the XML technology brings to the electronic commerce and the strategy used to extend the PRODNET infrastructure. The business processes in Virtual Enterprises are deeply dependent of communications among trading partners with heterogeneous technologies. The complexity of the exchanged messages requires a flexible, easy to understand, easy to manage and widely accepted representation language to model business information.
\end{abstract}

\section{INTRODUCTION}

There is a crescent consciousness of the entrepreneurial community about the need for a standard infrastructure able to run all the enterprise processes independently of the underlying technologies already in operation (legated) and also the emergent new ones presenting a significant added advantage to the enterprise business. The enterprises face a complex challenge considering that their installed technologies are not prepared to cope with the emergent electronic business to business (B2B) and/or the business to customer (B2C) global markets. Most of them have different subsystems in different technological stages and most of the times do not present an integrated perspective. The reality is made of a set of "automated islands" where interoperation (information exchange and information sharing among subsystems) is supported by handled processes or else by tailored process wrappers. This last technique is in fact widely used even if it generates, in most of the cases, integrated systems difficult to manage and maintain. Besides, considering its expensiveness, it is also out of the scope of the majority of the SMEs investment capacity.

\footnotetext{
"ISEL-DEEC, Electronics and Communication Engineering Department, $\{l o, m m b\} @ i s e l . p t$ The original version of this chapter was revised: The copyright line was incorrect. This has been corrected. The Erratum to this chapter is available at DOI: 10.1007/978-0-387-35399-9_52 
There is nowadays a trend pointing to a generalized effort to offer integrated platforms based on standards when they exist, or else, opening proprietary solutions making them look like standards to the market. This trend has however several drawbacks considering the conflicting market interests associated to such openness and standardization efforts. From this point of view, the world is divided into two main groups, one representing the target market (production logistics and services/management) and the other the technology industry. For the target market, standard solutions coping with the legated systems and enabling the adoption of the best technological solutions is, undoubtedly, the best of the worlds. If this would be possible, enterprises would be able select the best solutions without any technological constraint maintaining always an integrated, low cost, easy to maintain and scalable infrastructure.

Nevertheless, this is not the case and, for the next few years, even if some integration degree is expected to occur, non-standard systems will continue to populate the market. However the scenario is not so bad as the previous sentence would seems to present. In fact there are groups of standardization bodies/projects, governmental or not, working hard to achieve/propose an integrated view of the daily information and communication technologies. In some extent the situation is much more better that it was ten years ago. Important software companies are involved into such groups (IETF, W3C, OMG, WFMC, OAG, ISO, ITU, from many others) and the market is effectively using some of their results. As an example, a standard like the CORBA 2.3 published by the OMG group is getting a crescent importance with the growing number of products, implementing and relying on it.

At a different level, another emergent standard that is moving almost all the world, is the XML language and its related standards as DOM, XLS, from others, which are throwing the electronic commerce to a new dimension. The EDI/EDIFACT standard has played a major role in the classic B2B electronic commerce considering its main objective to establish a standardized representation for the exchange of electronic business message. It involves not only the normalization of the message format but also the semantics required to interpret message contents when data is moved among different information systems (different business applications).

The classic electronic commerce relies on three main requirements, the existence of computational systems, communication links and also intermediate message delivery entities, the Value Added Networks (VAN) offering enterprises a uniform and reliable delivery infrastructure (Gibon, 1999). Nevertheless the new challenges brought by the Internet technology suit and the Web infrastructure, are opening new opportunities to the electronic business. The Web infrastructure initially designed to offer nice information browsing capabilities with the HTML mark-up language, is now populated by a number of new features from direct data collection (CGI/forms), advertising, information search from many others.

The extensible mark-up language XML aims to establish a generic and flexible data representation format, based on meta-marks that can be formally defined into a file, the document type definition (DTD) that can be referenced from the XML document header. In opposition to the predefined HTML marks, XML marks are established by underlying data models defined by the DTD. This flexibility to represent complex data types is being used to exchange information among applications and to browse them in standard web browser. Its association with the 
efforts the EDI community has been doing to normalize semantics, is considered as a powerful mechanism to support the exchange of business documents among heterogeneous subsystems. An interesting application might involve the generation of a legal invoice to be printed at home as a consequence of a B2C purchase. This would greatly improve the electronic commerce considering that an authorized user can access and print, if necessary, an electronic document with legal value an important factor to establish the necessary trustiness on the underlying business framework.

Beyond a strategic discussion of the B2B and B2C under a virtual enterprise paradigm, this paper will present the main characteristics of the $\mathrm{XML}$ and related technologies considering its adoption as an added value to the EDI efforts to improve a new dimension for the electronic commerce. The XML language is expected to definitely contribute to a merging process between the $\mathrm{B} 2 \mathrm{C}$ and the classical B2B electronic commerce, the semantics worked by the EDI community and the data representation flexibility offered by XML are the basis for such trend. The adoption of XML will be discussed considering its adoption by the EDI module developed in the PRODNET project (Camarinha-Matos, 1999) and considering the added communication requirements established by this new business document format. This involves also the discussion of the implications for the PRODNET communication infrastructure, the PCI module (Osorio, 1999), in order to be able to cope with XML documents accessed from a Web browser or exchanged in the context of a virtual enterprise transaction.

\section{THE XML AND RELATED STANDARDS}

The Extensible Markup Language (XML) aims to store structured information in a readable text format. It is a subset of the Standard Generalized Markup Language (SGML) [ISO 8879], and a well-formed XML document is also conforming the SGML standard. The HTML is also conforming to the SGML language even if it is more restrictive than the XML. There are other standards that were considered as complementary of the XML standardization process like the Unicode and ISO/IEC 10646 for wide character representation. This makes the textual XML messages accessible from the different world spoken languages.

The XML has a structure very similar to the HTML language both are markup languages. The main difference is on the flexibility introduced by XML to enable the definition of new marks while the HTML is standardized with a predefined and a fixed set.

In fact the flexibility of the XML language relies on the ability to define marks whose meaning can be interpreted by humans. The meaningful of the mark's text are computationally interpreted with the help of a Document Type Definition (DTD) stored into referenced file or else embedded in the XML document itself.

The example presented in Figure 1, inspired from W3C recommendations (W3C, 1998), (W3C, 1999), shows a simplified representation of a book in well-formed XML.

The Figure 2, shows the content of the document type definition referenced by the XML book file. 


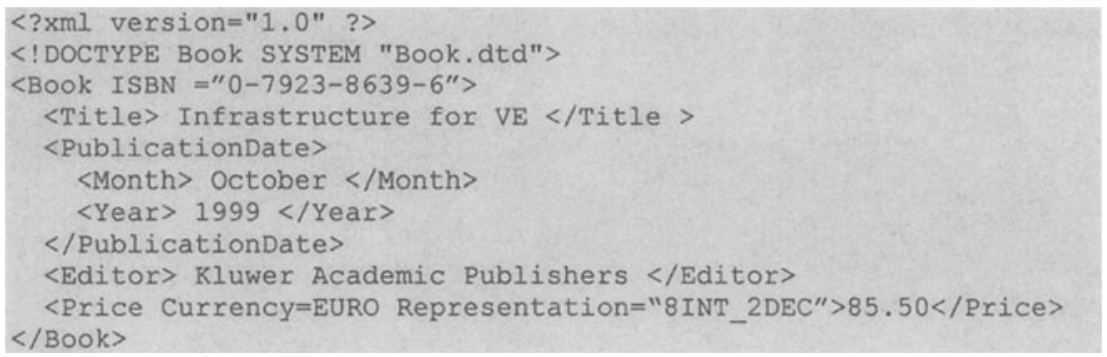

Figure 1 - A simplified book represented in XML

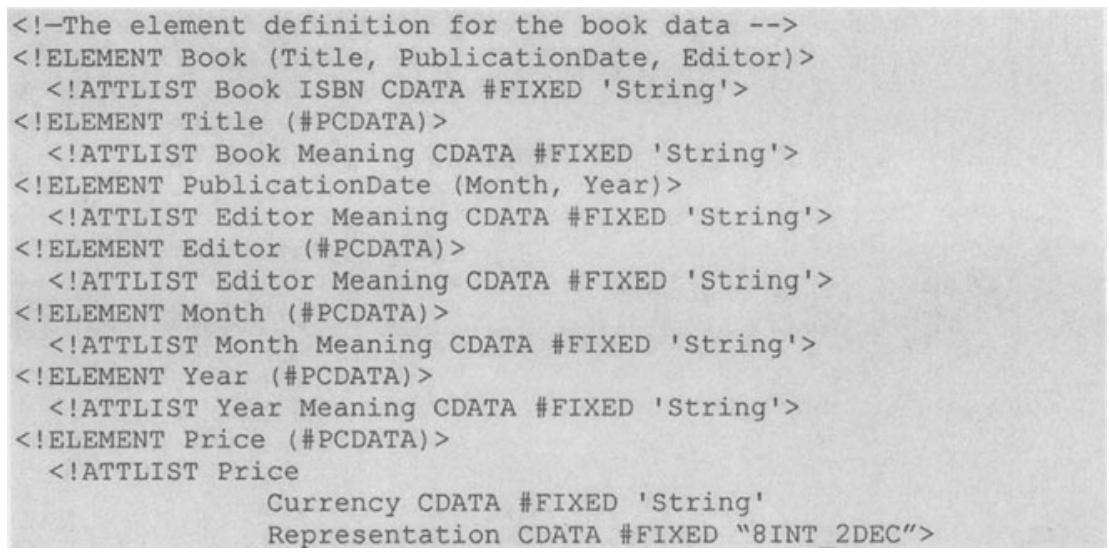

Figure 2 - The DTD content referenced in the XML book message

A XML document is organized around a sequence of elements. To be well formed, the root element must occur once as the first element into the document. The type of an element is identified by its name and it may have an associated set of attributes and a content. The content might range from empty, a simple string data value or it might be a complex element formed by a set of embedded elements. The XML elements have a direct correspondence to the HLML marks. It is however possible to write a XML file with any combination of elements (equivalents to the HTML marks), each one with any number of attached attributes and associated content. It is the responsibility of the user and/or an application program to interpret the XML file content, retrieving from it the meta-data according the XML grammar in order to check if the document is well formed. The verification of the wellformedness might involve a specification part that can be embedded into the document or as a reference to an external DTD file. A Document Type Definition (DTD) file embodies a set of XML declarations specifying the structure of the elements and the attributes that constitute a XML document instance. It is not mandatory to explicitly associate the document definition declarations to a document instance. However it is not possible to validate formally an XML document instance without such a DTD specification unless it is known a priori or else only if it is predefined as it happens with the HTML publishing language. 
The XML language has constructors to represent data types, complex or simple, offering a neutral textual representation able to be interpreted and integrated by different applications. It doesn't include directives to guide the presentation of the attached data, like it happens with the HTML. The generality of the XML language is contributing to ground a set of complementary initiatives aiming to establish a framework able to support a generalized exchange of information among subsystems including its presentation in a standard web browser. For data publishing the HTML, as part of the success of the web infrastructure, is moving to a more general presentation standard, the XHTML that is expected to succeed the HTML 4 (XHTML 1.0, 2000). For publishing there are however other more specialized standards like the Cascade Style Sheets (CSS) or a more recent and powerful language the Extensible Style Language (XSL).

Another interesting aspect is related with the extended behavior at the client side, when the information is browsed. In this case there are several proprietary script languages to attach or be referenced by an HTML documents like JavaScript, Java, VBScript and others. The Document Object Model (DOM) is a proposal of W3C consortium to create a common programming interface in order to maintain the portability of the information to be browsed, avoiding several trends to proprietary features only presentable without problems in a few browsers.

\section{SYNERGETIC JOINING BETWEEN THE EDI AND XML}

The EDI initiative has been a key contributor to the integration of heterogeneous applications, providing a standard textual representation for the exchange of business information/data. The target was to implement a mechanism to exchange data between applications running in different enterprises with heterogeneous underlying technologies, computational and communication ones. The lack of communication and computational standards has transformed the adoption of a generalized electronic exchange of documents among companies in a very complex task. Only in the 90's the EDI initiatives shifted from a more experimental period, started at the end of the 60's (Gibon, 1999), to a more generalized adoption of effective electronic exchange of documents. The UN-EDIFACT initiative was established in September 1986 and the first dictionary was published in 1990.

Based on the heterogeneity of the technology implemented by each trade partner, the EDI operation has been mainly supported by the Value Added Networks (VAN), see Figure 3. The VAN's offer a transparent and reliable message delivery that is implemented by flexible communication infrastructure and a complex mailbox management system.

The VAN entities are communication specialists that invested on a flexible communication infrastructure to exchange message packs among business partners. They offer the required Quality of Service (QoS) according the contracted communication service, giving to the enterprises the necessary trustiness in order to accomplish with the established business requirements. This business scenario was possible given the standardization efforts from several EDI initiatives. They developed a neutral representation for trading documents considering aspects from the character representation, data structure, organization and length and also the semantics associated to the exchanged data. 


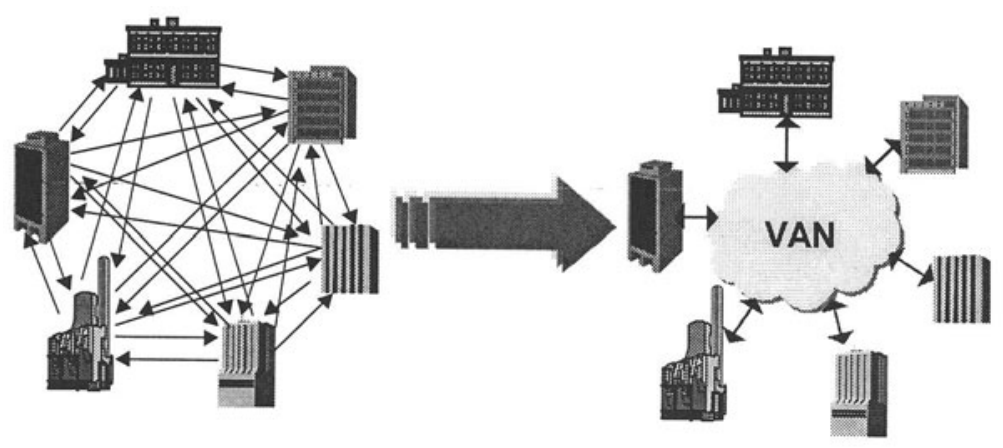

Figure 3 - The VAN EDI business model

The standardization promoted by the United Nations involving the United Nations Trade Data Interchange Directory (UNTDID) presents the following results:

- the EDIFACT syntax rules (based on the ISO 9735)

- Message design guidelines,

- Syntax Implementation guidelines,

- the EDIFACT Data Elements Directory,

- the EDIFACT Code List ,

- the EDIFACT Composite Data Elements Directory,

- the EDIFACT Standard Segments Directory,

- the EDIFACT United Nation Standard Messages Directory,

- Uniform Rules of Conduct for the Interchange of Trade Data by Teletransmission

This initiative involves different normalization process levels, ranging from physical data representation in a text file to a list of agreed identifiers (codes) with a generalized accepted semantics.

Even if a standard was adopted, the implementation level of EDI processes are nowadays centered around large companies and only in small companies when they are part of the value chain of those large companies. The automotive industry and the large distribution groups are paradigmatic examples of this status quo. In fact, the implementation of EDI processes has some complexity and it is considerably expensive, considering the amount of services needed to implement the interfaces between the enterprise data warehouses/applications and the VANs.

There is another aspect that has contributed to a weak adoption of the EDI technology. The available standards are difficult to implement, not only the software to generate and parse EDI/EDIFACT messages but, also, the large number of literal coding the semantics of the message content, using a few characters without any direct meaning for a person. The EDIFACT and the X.12 coded messages are not easily readable by persons what make them complex to handle by others than computer processes. Some products were developed to help message developers to access a friendlier tool to map the enterprise data to be used in EDI message generation to the EDIFACT (or X.12) format. The VisualCommerce developed by ILLICOM is an example of such a tool. It presents CASE facilities by offering a set 
of mechanisms, as a user friend interface to generate message exchanges by simple "drag and drop" operations.

Even considering the crescent flexibility of the EDI tools, the electronic business to business commerce continues confined to large enterprises with enough investment capacity to cope with the relative EDI business implementation complexity. There is however a new trend to get advantage from the Internet technology suit to speedup a generalized adoption of different forms of electronic business. The costs associated to the implementation of Internet based technological solutions are lower. Some of the required components are free or included in the basic configurations of different operating systems. As an example, the implementation of an Internet electronic mail system (SMTP/POP3) is incomparably less expensive and complex than a X.400 one. These cost factors are contributing to generalize the implementation of Internet accesses in a growing number of SMEs. Even if in most of the cases only e-mail and Web publishing is available, the technology is getting an increased interest with special importance to the increasing investments of the new and already established telecommunication companies. This democratization process promoted by the Internet technology suit are contributing to an increasing investment from the Internet Service Providers (ISP) with a crescent communication quality and also a crescent number of services, scaled according the offered QoS. Some ISP are starting to offer reliable accesses, with a predefined and configured set of mailboxes, with delivery services guaranteeing to the trading partners the quality they require for their electronic interchange processes.

This mass movement to the Internet arena is also affecting the EDI initiatives. The classical electronic commerce based on the classical VANs is moving in the direction of the Internet technologies taking advantage of the lower costs required to implement integration with standards widely accepted/de facto. Important normalization bodies centered in other domains include also working groups addressing the specificity of the EDI processes. The Object Management Group (OMG) is an example considering that it has a group addressing the electronic commerce where EDI represents an important role. The EDI process, as it looks nowadays, requires the integration with the most relevant initiative concerning the establishment of an integrating infrastructure logically connecting all the enterprise subsystems. This is a complex subject considering that it is not realistic a fast shift from the situation as it is, to a "nice" infrastructure where all components interact according some planned logic. Nevertheless, the Internet avalanche is forcing different legated technologies to redefine their strategy to cope with this crescent vague.

The XML emerges from such key technologies as a contributor to the redefinition of the classical EDI. In fact the XML presents some significant advantages against the utilization of the EDIFACT and X.12 used standards. The Table 1 shows different issues establishing a parallel between the utilization of EDI based on EDIFACT with the utilization of EDI based on XML.

The associated powerful to the XML language flexibility to represent different organization business data in a textual representation, is provoking innovative changes to the classical EDI. With EDIFACT a compact codification is used to achieve a neutral representation of the exchanged business documents. 
Table 1 - Comparing EDIFACT EDI with the EDI based on XML

\begin{tabular}{|l|l|}
\hline \multicolumn{1}{|c|}{ EDI based on EDIFACT } & \multicolumn{1}{|c|}{ EDI based on XML } \\
\hline $\begin{array}{l}\text { Language thought for very small in size. } \\
\text { Marks are short/codified for computer } \\
\text { processing }\end{array}$ & $\begin{array}{l}\text { The language was designed to generate human } \\
\text { readable messages, easy to generate }\end{array}$ \\
\hline $\begin{array}{l}\text { Programming of parsers and message } \\
\text { generators are complex. It requires a } \\
\text { programming language like C++, } \\
\text { Delphi/Pascal or other }\end{array}$ & $\begin{array}{l}\text { Easy to generate parsers and message } \\
\text { generators. JavaScript, Perl or other script } \\
\text { language is enough. }\end{array}$ \\
\hline $\begin{array}{l}\text { The generation of new messages may take } \\
\text { months to prepare. The access to } \\
\text { specialized CASE tools might reduce this } \\
\text { period. }\end{array}$ & $\begin{array}{l}\text { Messages are simpler to generate considering } \\
\text { its soundness (long and clear identifiers), a } \\
\text { new message may spend hours to prepare. The } \\
\text { CASE tools might contribute to facilitate the } \\
\text { message creation. }\end{array}$ \\
\hline $\begin{array}{l}\text { The message information is limited to a } \\
\text { reduced set of characters. }\end{array}$ & $\begin{array}{l}\text { Processor instructions and other information } \\
\text { components (images, drawing instructions) can } \\
\text { be included (XSL, Xforms, others). }\end{array}$ \\
\hline $\begin{array}{l}\text { EDIFACT stream character data is limited } \\
\text { to eight bit char representation }\end{array}$ & $\begin{array}{l}\text { The representation of the message involves } \\
\text { wide chars including Unicode, supporting } \\
\text { different languages }\end{array}$ \\
\hline
\end{tabular}

The XML language makes possible the representation of the same entities represented in EDIFACT or X.12 but under a user friendlier format. One important aspect that guided the design of the XML language was its friendliness considering the message design and its interpretation by any of the involved persons. On the other hand the design of the EDIFACT technology was guided by the expensiveness of disk space and communication costs (when measured in message size). Therefore, it was designed as a compact language difficult to read by humans and also difficult to generate considering its unmeaning coded marks.

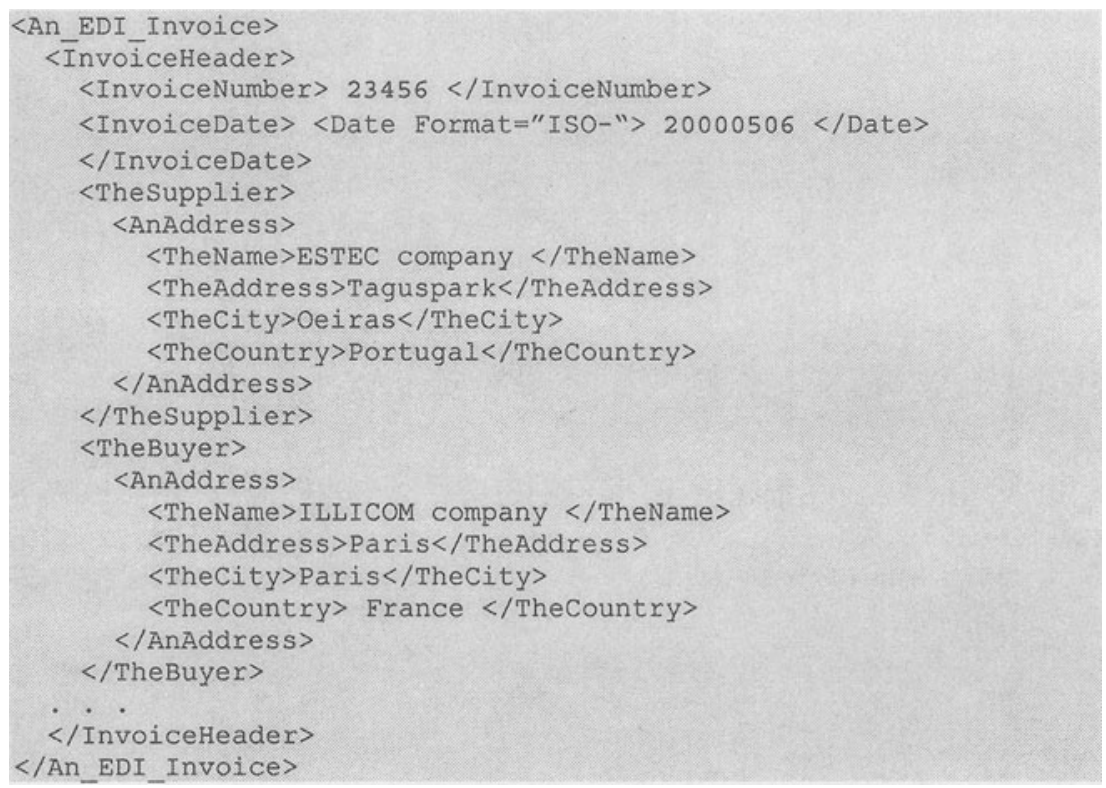

Figure 4 - An example of a simple XML message 
Considering that nowadays, the memory resources are cheaper and that communication is getting a decreasing weight on all business messages exchange cycle, the focuses is now on the simplicity of both message development and user accessibility to its content (interpretation).

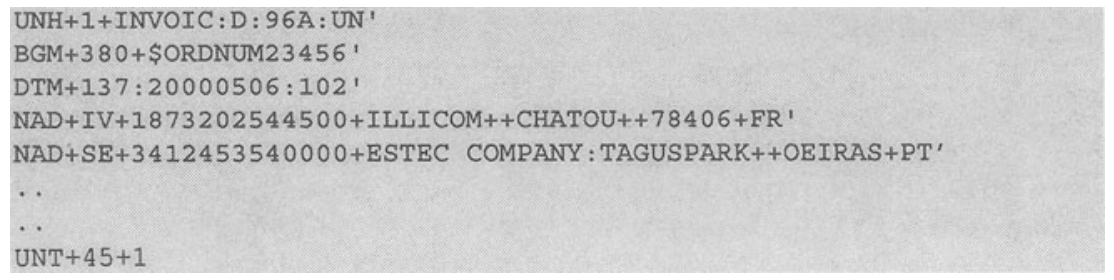

Figure 5 - The same example in EDIFACT

An EDIFACT message is difficult to be interpreted by others than those experts involved in the message design, while for a XML message any person can easily interpret it. The interpretation of an EDIFACT message requires the some knowledge about the standard, while the symbolic nature of the XML language facilitates its interpretation even for people that do not learned details about the XML language. It is important to make clear that XML promotes this clearness, but it is only effective if XML components are defined under this principle. It is possible to write an unclear XML message by using codified (short and meaningless) identifiers.

\section{TRANSPORTING SEMANTICS FROM EDIFACT TO EDI- XML}

The XML language presents a significant number of advantages to the EDI community. Some of the advantages are:

- EDI-XML messages are human readable what make them more manageable/accessible

- The XML language is based on elements that can embed other elements as content. Each element can have an associated set of attributes. The elements have an associated identifier and a type used to establish language marks

- The XML as it happens with HTML, derives from the standard SGML and is being used to ground other specifications. It is becoming a generalized and flexible modeling language to specify other Internet languages/protocols. The Single Object Access Protocol (SOAP) (IETFSOAP, 1999) that uses XML as the language to implement the remote procedure calls tunneled though HTTP, is an example of the general utilization of the XML language

Complementary standards like XSL used to guide the presentation of an XML file in a standard Web browser, are being developed 
Definitely the XML language is being established as a generalized language to model data in different contexts. Several tools are being developed to support different functions from message design, mapping between other neutral file formats to XML and the reverse operations.

Nevertheless, the XML doesn't solve all the existing problems by offering enterprises a mechanism to integrate all the subsystems including a transparent message exchange among them. Each enterprise has its own technological and organizational culture, its own information system, integrated or organized around a set of subsystems in different technological stages. Therefore, the connection of an enterprise to its trading partners is not an easy task. There are several factors contributing to offer Internet as an infrastructure to ground electronic commerce among enterprises:

- some ISP's are offering transparent mechanisms to deliver messages to any address

- some enterprise are able to cope with the low QoS level offered by the Internet infrastructure, considering the specificity of the underlying processes

- there are technological infrastructure able to offer the required QoS by implementing mechanisms to make transparent some of the Internet weaknesses. The PRODNET communication infrastructure does (Osório, 1999) is an example of such infrastructure.

Beyond the technological infrastructure it is also necessary to integrate the efforts made on semantics normalization under the EDIFACT project, integrating them into the EDI-XML framework. It is not enough to establish nice XML schemas defined by DTD files, it is also necessary to establish a standard for the meaning of each element value in the XML files exchanged among trading partner. The ebXML project is an important initiative in this direction it "aims to provide an open technical framework to enable XML to be utilized in a consistent and uniform manner for the exchange of Electronic Business (EB) data in application to application, application to human and human to application environments thus creating a single global market' (Rawlins, 2000). The ebXML project joins the United Nations Center for the Facilitation of Procedures and Practices for Administration, Commerce and Transport (UN/CEFACT) and the Organization for the Advancement of Structured Information Standards (OASIS). This and other related projects are unanimous about the advantage to import the existing EDI efforts on semantics in order to they contribute to create a repository of XML elements/attributes based on UN/EDIFACT standard.

\section{ADDING XML TO THE PRODNET INFRASTRUCTURE}

The PRODNET general architecture (Camarinha-Matos, 1999), (Hafsarmanesh, 1999) Figure 6, involves two main parts: the internal module representing the enterprise applications and the cooperation layer grouping a set of modules implementing cooperation activities on the behalf of a workflow plan executed by the Local Coordinator Module (LCM) (Camarinha, 1999). 


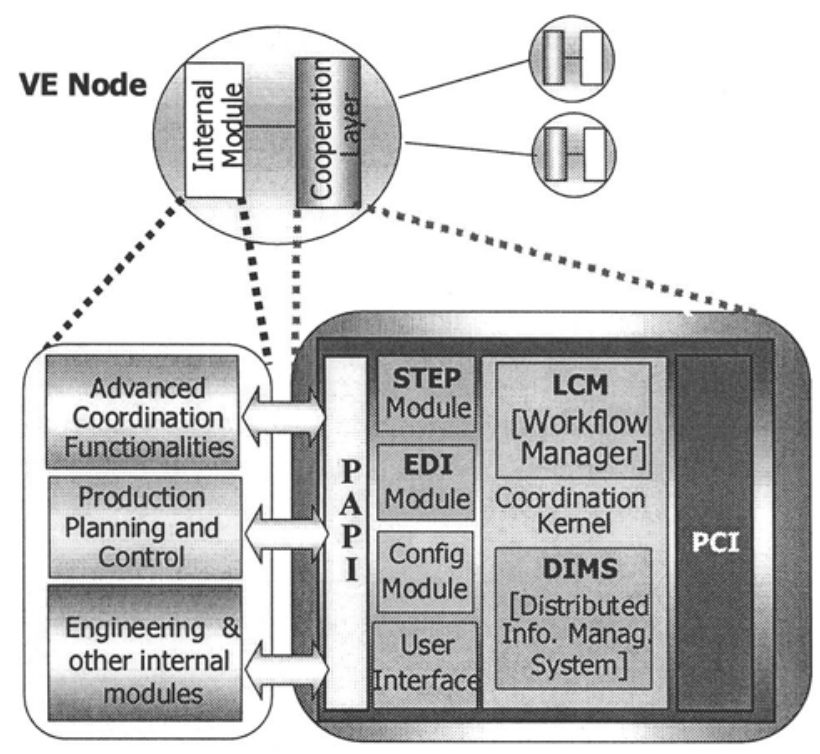

Figure 6 - The PRODNET general architecture

The applications belonging to the internal module, like those applications supporting Enterprise Resource Planning (ERP), product development, management and others, access Core Services (Camarinha-Matos, 1999) exported by the cooperation layer. Each Core Services represents a different workflow process (plan) guiding all the steps necessary to manage exchanged messages in the context of a virtual enterprise. According a specific Core Service the cooperation layer processes the message to be exchanged by calling the required modules to process it. For an EDI message, the core service involves the EDI module to generate, for each planned exchange, the respective message in the EDIFACT format. The same module parses EDI messages received from other VE members.

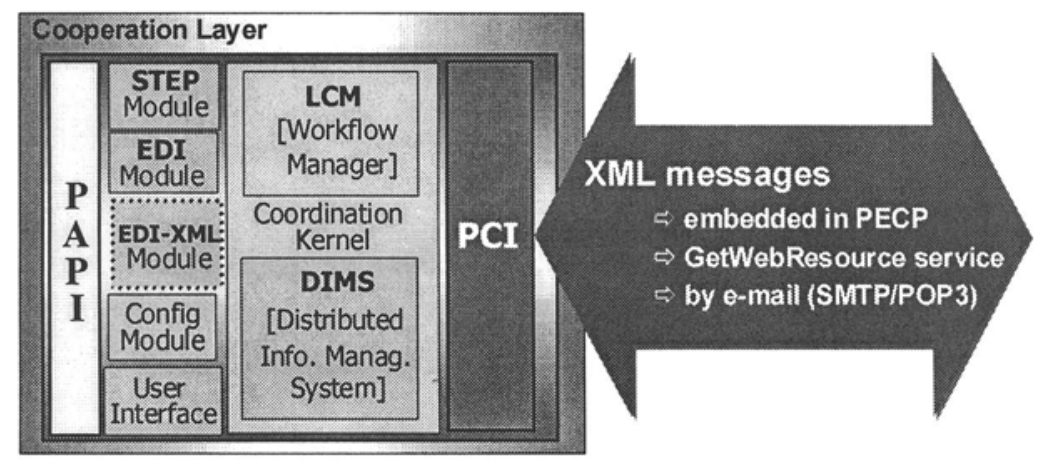

Figure 7 - The PRODNET Cooperation Layer enhanced with the EDI-XML module 
To deal with EDI-XML messages, whose content is a well-formed XML content, it is required a new service module specialized in processing/parsing XML messages or else the development of a new version of the EDI module supporting the XML standard.

The new EDI-XML service might need to access a URI resource when referenced inside an XML message by an URL address, as shown in the example below.

$<! D O C T Y P E$ spec PUBLIC "http://www.EDI-Dictionary.org/XML/book.dtd">

A hypothetical www.EDI-Dictionary.org web server stores the DTD definition to be generally accessed when some trade partner has to process an EDI-XML data file.

In this case, the EDI-XML service needs to access via HTTP the www.EDIDictionary.org server to get from it the file book.dtd, if it is not yet cached from an earlier access to the same definition file. The XML framework, as it is being conceived groups around it a number of complementary standards but not yet completely defined. However, the basic XML philosophy points to the requirement of a flexible access to complementary definition files, be them DTD's, the emergent schema definitions, style sheet information or other complementary information to guide specialized processes, like browsing, printing and others.

This flexibility to access complementary information attached to a XML message requires from the PRODNET Communication Infrastructure (PCI) (Osório, 1999) an extended set of services to offer a secure access to such referenced resources.

The PCI already offers integration with the Web infrastructure through the CGI infrastructure (Osório 2000). However, a new communication resource based on the HTTP transport protocol needs to be integrated into the PCI infrastructure. This way, the PCI communication manager is able to get resources from the Web by establishing HTTT or SHTTP (if a secure access is required) connections, Figure 8.

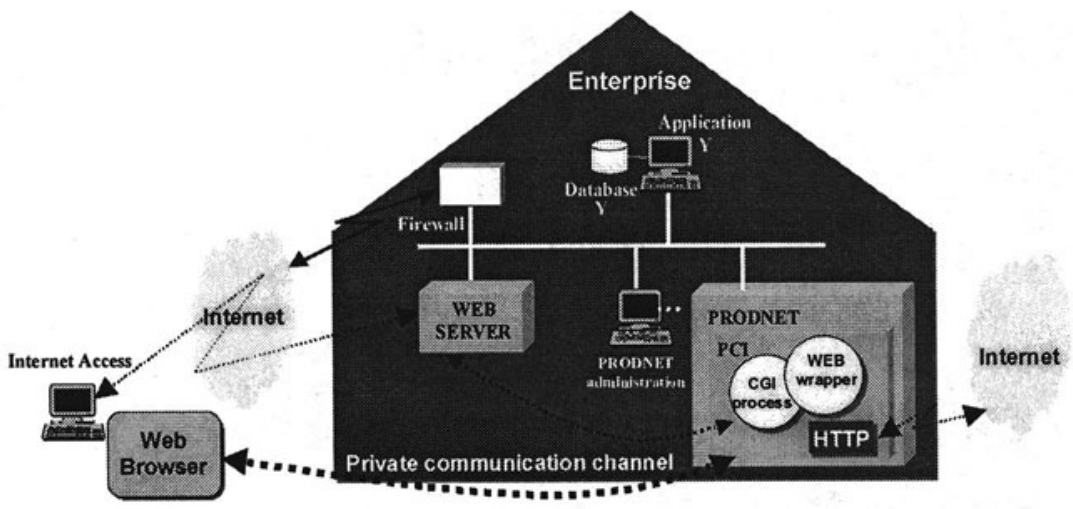

Figure 8 - Web services of the PRODNET Communication Infrastructure

The secure access involves the Transport Layer Security (TLS) standard (known better as its predecessor Secure Socket Layer-SSL 3.0) (RFC-2246, 1999) used to implement secure communications between web servers and client web browsers. 
The TLS aims primarily to provide privacy and data integrity between process peers, nevertheless TLS includes also a Record Protocol used to encapsulate higher level protocols like the TLS Handshake Protocol, aiming to negotiate authentication based on digital certificates.

This added features extend the PCI facilities on the track of the established strategy during the PCI architecture definition: "adoption as much as possible of standards to wideness PCI interoperability" (Osorio, 1997). This perspective makes possible the implementation of extended services like the new GetWebResource aiming to retrieve from a web DTD file or any other resource see Figure 7. The access implemented by this new service might use a clear or a secure and authenticated connection depending on the quality of services specified in service parameters (Osório, 1999).

\section{CONCLUSIONS}

The XML language and the related proposals and initiatives to developed this technology suit are at an initial stage. There is a market consensus that XML will play a major role in systems integration. The initiatives that most of the well positioned companies have in XML domain and related technologies points to this direction.

This paper stressed however that XML is not alone a solution for all the interoperability problems. The EDI initiatives mainly those under the United Nations sponsorship like the UN/EDIFACT standard, are expected to bring their efforts, done along the last decade to help to establish a precise semantics for different business messages in a diversified set of industrial sectors.

Following the presentation of some general XML trends, this paper presented a strategy to enhance the PRODNET infrastructure to cope with XML framework. It was proposed to extend the Cooperation Layer functionality with XML message handling. A new EDI-XML module or else the required new functions implemented by the existing EDI module were proposed. This extension involves also the PRODNET Communication Infrastructure for which it was proposed the integration of a new communication resource to operate with an HTTP connection. In order to cope with Web security and authenticated connections the SSL/TLS and complementary protocols were considered.

It is mandatory for any communication infrastructure to be aware about the emergent standards. A generalized interoperability among applications or subsystems requires an agreement on a set of commonly accepted standards. The strategy here proposed involves also a permanent awareness not only about technology but also about the understandings of the electronic commerce policies. This involves not only normalization of messages structure and semantics but also security policies from other aspects.

\section{ACKNOWLEDGEMENTS}

This work was funded in part by the European Commission, Esprit programme within the PRODNET II project. The authors also thank the valuable contributions 
from the consortium partners: ILLICOM (F), Universidade Nova de Lisboa (P), University of Amsterdam (NL), Universidade Federal de Santa Catarina (BR), Uninova (P), CSIN (P), HERTEN (BR), ProSTEP (D), MIRALAGO (P).

\section{REFERENCES}

1. Afsarmanesh, H., Garita C., Ugur Y., Frenkel A., Hertzberger L. O. (1999) Federated Information Management Requirements for Virtual Enterprise, Kluwer Academic Publishers, ISBN 0-79238639-6, cap. 3 part I, pp. 31-48.

2. Camarinha-Matos, L. M., Afsarmanesh, H. (1999) Tendencies and General Requirements for Virtual Enterprise, Kluwer Academic Publishers, ISBN 0-7923-8639-6, cap. 2 part I, pp. 15-30.

3. Camarinha-Matos, L. M., Afsarmanesh, H. (1999) The Virtual Enterprise Concept ${ }_{2}$ Kluwer Academic Publishers, ISBN 0-7923-8639-6, cap. 1 part I, pp. 3-14,

4. Camarinha-Matos, L. M., Lima, C. (1999) Coordination and Configuration Requirements in a Virtual Enterprise, Kluwer Academic Publishers, ISBN 0-7923-8639-6, cap. 4 part I, pp. 49-64.

5. Gibon P. Clavier J. F., Loison S., (1999) Support for Electronic data Interchange, Kluwer Publishers, ISBN 0-7923-8639-6, cap. 5 part I, pp. 187-208.

6. IETF SOAP (1999) Simple Object Access Protocol a IETF draft standard, November.

7 Osório A. L., Barata M. M. (2000) Reliable and Secure Communications Infrastructure for Virtual Enterprises, to be published in the Industrial Automation magazine during 2000.

8. Osório A. L., Barata M. M., Gibon P. (1999) Communication Infrastructure Requirement in a Virtual Enterprise, in the book "Infrastructure for Virtual Enterprises" edited by Camarinha-Matos L.M and Hamideh Afsarmanesh, Kluwer Publishers, ISBN 0-7923-8639-6, cap. 5 part I, pp. 65-76.

9. Osório A. L.,Antunes C., Barata M. M. (1999) The PRODNET Communication Infrastructure, Kluwer Publishers, ISBN 0-7923-8639-6, cap. 11 part II, pp. 167-186.

10. Osório, A. Luís; Gibon, Pierre; Barata M. Martins (1998) Secure Electronic Commerce in Virtual Enterprises of SMEs, presented at the international conference BASYS'98, 27-28 August 1998 in Prague and published by Kluwer.

11. Rawlins M., Crawford M. (2000) electronic business XML (ebXML) Requirements Specification, from www.ebxml.org.

12. RFC-2246 (1999) Transport Layer Security version 1.0, an Internet Official Protocol Standards (STD 1), January 1999.

13. Schneier B. (1996) Applied Cryptography. 2nd edition, John Wiley \& Sons.

14. W3C (1998) Extensible Markup Language (XML) 1.0, REC-xml-19980210, W3C Recommendation 10-February-1998.

15. W3C (1999) Namespaces in XML, recommendation REC-xml-names-19990114

16. World Wide Web Consortium 14-January-1999.

17. XHTML ${ }^{\mathrm{TM}} 1.0(2000)$ The Extensible HyperText Markup Language, a Reformulation of HTML 4 in XML 1.0, a W3C Recommendation 26 January 2000. 